\title{
Parallel algorithm of a modified surface modeling method and its application in digital elevation model construction
}

\author{
Mingwei Zhao $\cdot$ Tianxiang Yue $\cdot$ Na Zhao $\cdot$ \\ Xin Yang $\cdot$ Yifu Wang $\cdot$ Xingying Zhang
}

Received: 7 January 2015/Accepted: 10 February 2015/Published online: 4 March 2015

(C) Springer-Verlag Berlin Heidelberg 2015

\begin{abstract}
High accuracy surface modeling (HASM) has been proved to be a superior method for surface simulation compared to classical interpolation methods. However, the fact that HASM is time consuming combined with its dependence on its driving field restricts its application in large area problems. This research develops a modified HASM which can get rid of the driving field in the surface simulation, and the parallel version of the modified HASM is also proposed with the purpose of improving its computational efficiency. Light detection and ranging (LIDAR) data are used as an optimum constraint to construct digital elevation model (DEM). Tests show that the modified HASM can perform surface simulation successfully without the driving field. And it also shows that the simulation accuracy of the modified HASM is almost the same as the old HASM and the classical interpolation methods when the sampling rate is larger than $0.5 \%$, while the modified HASM shows significantly increased simulation accuracy as the sampling rate
\end{abstract}

M. Zhao · T. Yue $(\bowtie) \cdot N$. Zhao · Y. Wang

State Key Laboratory of Resources and Environment Information System, Institute of Geographic Sciences and Natural Resources Research, CAS, Beijing 10010, China e-mail: yue@lreis.ac.cn

M. Zhao

e-mail: zhaomw@1reis.ac.cn

M. Zhao · Y. Wang

University of Chinese Academy of Sciences,

Beijing 100049, China

M. Zhao $\cdot$ X. Zhang

National Satellite Meteorological Center, China Meteorological Administration, Beijing 100081, China

X. Yang

University of Jinan Quancheng College, Penglai 265600, China decreases. This characteristic indicates that the modified HASM no longer relies on the driving field in the surface simulation. And it also improves the simulation accuracy compared to the old HASM and the classical interpolation methods. Tests of parallel efficiency show that the masterslave mode used in the parallel algorithm obtains a satisfactory result, indicating that the HASM can be applied to surface simulation of large area problems. And it also shows that the modified HASM would have great potential where applied in high-resolution DEM and digital surface model (DSM) construction from LIDAR data.

Keywords HASM - DEM - LIDAR · Parallel algorithm · Simulation accuracy

\section{Introduction}

Geomorphology, the study of landscape change, thus stands in the center of Earth surface science, where strong couplings link human dynamics, biology, biochemistry, geochemistry, geology, hydrology, and atmospheric dynamics, including climate change (Murray et al. 2009). Information on the Earth's relief is a key parameter for almost any geoscientific analysis and for all precise land-oriented applications and planning purposes (Dech 2005). Understanding earth surface processes relies on modern digital terrain representations and depends strongly on the quality of topographic data (Tarolli et al. 2009; Tang et al. 2013; Alkhasawneh et al. 2014; Gupta et al. 2014). Terrestrial laser scanner (TLS) and airborne laser swath mapping technology (ALSM) are able to produce sub-meter resolution digital terrain models and high-quality land cover information over large areas. The shuttle radar topographic mission (SRTM) and the advanced space borne thermal emission and 
reflection radiometer (ASTER) have allowed detailed analyses in large regions through their DEMs products.

The Earth's surface obeys the conditions of uniqueness, continuity, smoothness, and finiteness because the Earth's surface height is restricted in space by the value of gravity and cannot be infinitely large or infinitely small. Attributes of the Earth's surface for special consideration where it comes to surface modeling include lines of relief discontinuity, steep and overhanging scarps, acute peaks, niches, caves, karstpits and sinkholes, as well as other elements violating the condition of smoothness. The shape of the Earth's surface was firstly expressed by the means of gravity action at ground level (De Gbaaff-Hunter 1937). The shape of the topographical earth surface was calculated by means of gravity measurements on that surface by solving two integral equations (Bragard 1965). The possibility of constructing potential expansions, which would converge on the Earth's surface, was explored by generalizing the external expansion of the Earth's potential in spherical harmonics to the Earth's surface (Petrovskaya 1977), and the so-called spherical harmonics are the angular portion of a set of solutions to Laplace's equation in mathematics.

A nonlinear descending principle was developed based on the theory of molecular mechanics of rock to calculate the altitude's limit on the Earth's surface (Niu 1993). An F-approximation method and an S-approximation method were developed to simulate the Earth's surface topography. The F-approximation of earth surface topography was based on the Strakhov method of linear integral representations, and represented by means of the Fourier integral (Strakhov et al. 1999). The S-approximation was based on the fundamental formula of the theory of harmonic functions and specified by means of the Poisson integral (Stepanova 2007). Harmonic functions were employed to simulate the Earth's surface where displacements took place and can be picked up by global positioning system (GPS) measurements (Ionescu and Volkov 2008).

It is proven that the equation of the Earth's surface can be formulated as (Kerimov, 2009), $z=f(x, y)$ where $z$ is an attribute value of the Earth's surface at location $(x, y)$. For the surface $z=f(x, y)$, an iterative formulation of HASM was developed in terms of the fundamental theorem for surfaces (Yue et al. 2007), which was transformed into a symmetric positive-definite and large sparse linear system. HASM has a huge computation cost because it must use an equation set for simulating each lattice of a surface. To speed up the computation of HASM, we developed a multigrid method of HASM (HASM-MG) (Yue and Song 2008), an adaptive method of HASM (HASM-AM) (Yue et al. 2010a), an adjustment computation of HASM (HASM-AC) (Yue and Wang 2010), and a preconditioned conjugate gradient (PCG) algorithm of HASM (HASMPCG) (Yue et al. 2010b). The multigrid method is the fastest numerical method for solving partial differential equations, based on the two principles of error smoothing and coarse grid correction. The principle of the adaptive method is that grid cells where the error is large will be marked for refinement, while satisfactorily accurate grid cells are left unchanged. The adjustment computation permits all observations, regardless of their number or type, to be entered into the adjustment and used simultaneously in the computations by means of least squares. A conjugate gradient algorithm was originally viewed as an acceleration technique for the effective solution of large linear systems by a succession of well-convergent approximations; the preconditioned conjugate gradient algorithm being developed by introducing a precondition to ensure faster convergence of the conjugate gradient method.

However, HASM also has some imperfections, including two serious defects; the first being that HASM requires a driving field when it performs a surface simulation, which is generated by other spatial interpolation methods, such as inverse distance weighted (IDW) and Kriging. This demand for a driving field simultaneously complicates HASM and makes it more time consuming; time consumption being HASM's second key defect (Zhao et al. 2014a). Though many HASM algorithms have been put forward, most of these algorithms mainly focus on the improving simulation accuracy, and yet low simulation efficiency has not yet been sufficiently addressed. These defects mean that HASM cannot perform large area and high-resolution surface simulations, making HASM difficult to apply to practical surface simulation.

To address these problems in HASM, this paper puts forward various solutions. To solve HASM's dependence on the driving field, the third Gaussian equation is brought to the HASM and a modified HASM which contains three equations is constructed. For the sake of improving the simulation efficiency, this research designs a master-slave mode parallel algorithm based on data segmentation strategy. The paper is structured as follows: The first section introduces the development of HASM and its application in DEM construction, and then lists the main defects of HASM as well as putting forward the main aims of this paper. The second section introduces the test area and test data. The third section describes the modified HASM in detail and its parallelization. The forth section analyses increased simulation accuracy of the modified HASM and the parallel efficiency. The last section draws conclusion based on this paper's aims and explores possible avenues for further research.

\section{Study area and data}

The study area is located in Tianlao Pool basin $\left(38^{\circ} 23^{\prime} 55^{\prime \prime} \sim 38^{\circ} 26^{\prime} 57^{\prime \prime} \mathrm{N}, 99^{\circ} 53^{\prime} 45^{\prime \prime} \sim 99^{\circ} 57^{\prime} 12^{\prime \prime} \mathrm{E}\right)$. Tianlao 
Pool basin (Fig. 1), upstream of the Hei River is part of the Qilian Mountain, and its elevation varies from 2600 to $4400 \mathrm{~m}$. Tianlao Pool basin belongs to alpine Semi-arid mountain forest steppe climate, the annual average temperature is about $0.6{ }^{\circ} \mathrm{C}$, the annual precipitation is $437.2 \mathrm{~mm}$, the evaporation capacity is $1066 \mathrm{~mm}$, and the annual average relative humidity is $59 \%$ (Liang et al. 2013). The soil and vegetation types show clear variation along with the differences of mountainous topography and climate, and both form an obvious vertical distribution belt.

The Lidar data were produced on July 25, 2012. The Lidar instrument is the ALS70LIDAR system made by Leica and the carrying platform is $\mathrm{Y}-12$. The wave length of the ALS70LIDAR system is $1064 \mathrm{~nm}$. The density of the Lidar points is approximately $1 / \mathrm{m}^{2}$. The Lidar points are divided into ground points, vegetation points and other points through several processing steps, of which the ground points are used to generate DEMs in this study. A square area $(2.5 \mathrm{~km} \times 2.5 \mathrm{~km})$ in the central part of the Tianlao Pool is chosen to test the efficiency of the parallel algorithm, while ten small sub-areas are selected in the study area to test the accuracy and time consuming of the method (Fig. 1).

\section{Methodology}

In this section, the modified HASM will be introduced and the main steps of this model in surface simulation are listed. Then, the parallel version of the modified HASM is discussed.

\section{HASM and its modification}

A surface is determined by its first and the second fundamental coefficients based on differential geometry (Henderson 1998). Suppose a surface can be expressed as $z=f(x, y)$, the first fundamental coefficients $E, F, G$ can be expressed as formula (3.1), and the second fundamental coefficients $L, M, N$ can be expressed as formula (3.2).

$$
\begin{aligned}
& E=1+f_{\mathrm{x}}^{2}, F=f_{\mathrm{x}} \cdot f_{\mathrm{y}}, G=1+f_{\mathrm{y}}^{2} \\
& L=\frac{f_{\mathrm{xx}}}{\sqrt{1+f_{\mathrm{x}}^{2}+f_{\mathrm{y}}^{2}}}, \\
& M=\frac{f_{\mathrm{xy}}}{\sqrt{1+f_{\mathrm{x}}^{2}+f_{\mathrm{y}}^{2}}}, N=\frac{f_{\mathrm{yy}}}{\sqrt{1+f_{\mathrm{x}}^{2}+f_{\mathrm{y}}^{2}}}
\end{aligned}
$$

where $f_{\mathrm{x}}$ represents the first-order partial derivative of the function $z=f(x, y)$ with respect to $x, f_{\mathrm{y}}$ represents the firstorder partial derivative with respect toy, $f_{\mathrm{xx}}$ represents the second-order partial derivative with respect to $x, f_{\mathrm{yy}}$ represents the second-order partial derivative with respect to $y$, and $f_{\mathrm{xy}}$ represents the mixed partial derivative first with respect to $x$ and then with respect to $y$. The entire derivative can be calculated through finite difference methods (Zhao et al. 2014a).

Partial differential equations of the surface theory require $E, F, G, L, M, N$ which must satisfy the following Gauss equations (Somasundaram 2005; Toponogov 2006; Yue 2011):

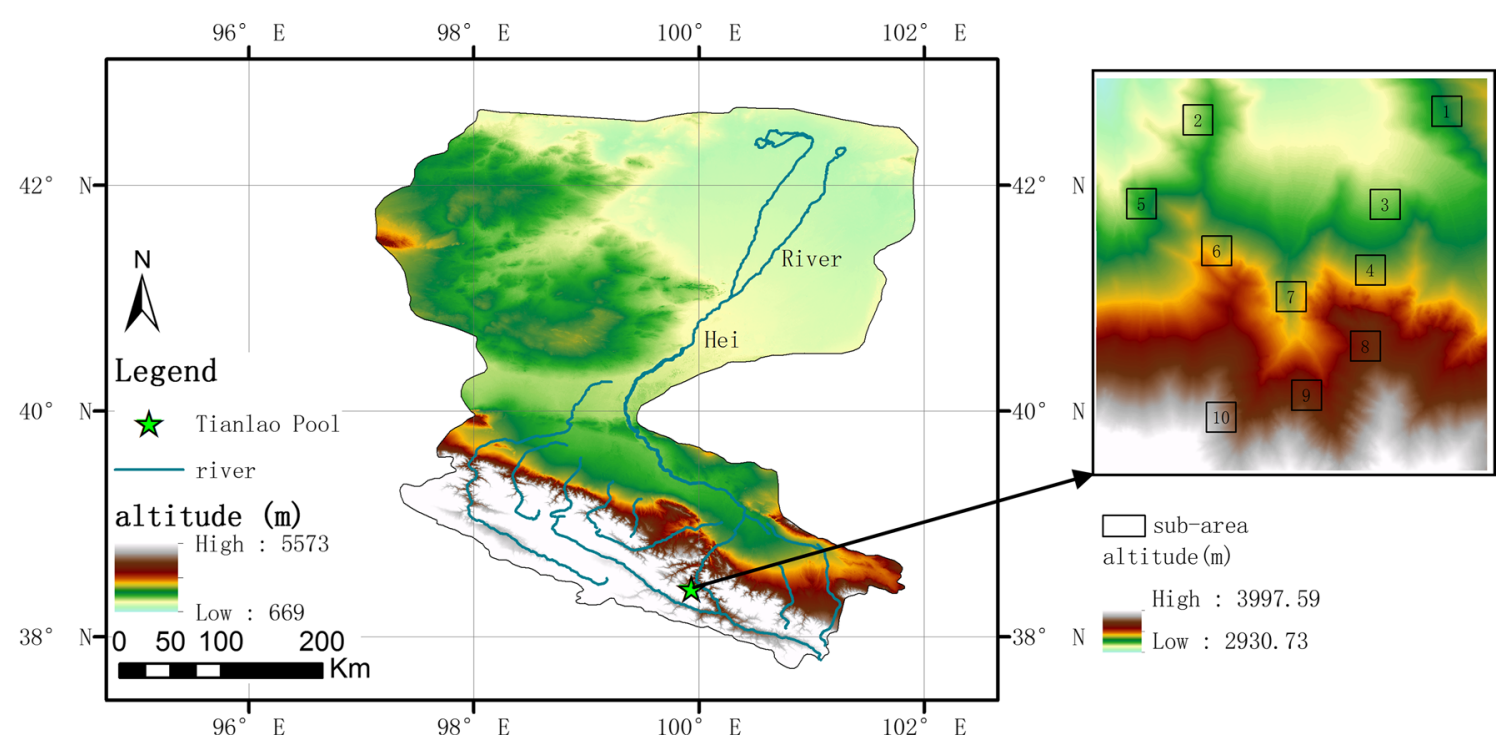

Fig. 1 Geographical location of the test area and the test sub-areas in Hei River Basin 
$\left\{\begin{array}{l}f_{\mathrm{xx}}=\Gamma_{11}^{1} f_{\mathrm{x}}+\Gamma_{11}^{2} f_{\mathrm{y}}+\frac{L}{\sqrt{E+G-1}} \\ f_{\mathrm{yy}}=\Gamma_{22}^{1} f_{\mathrm{x}}+\Gamma_{22}^{2} f_{\mathrm{y}}+\frac{N}{\sqrt{E+G-1}} \\ f_{\mathrm{xy}}=\Gamma_{12}^{1} f_{\mathrm{x}}+\Gamma_{12}^{2} f_{\mathrm{y}}+\frac{M}{\sqrt{E+G-1}}\end{array}\right.$

In which, $\Gamma_{11}^{1}=\frac{\mathrm{GE}_{\mathrm{x}}-2 \mathrm{FF}_{\mathrm{x}}+\mathrm{FE}_{\mathrm{y}}}{2\left(\mathrm{EG}-F^{2}\right)}, \Gamma_{11}^{2}=\frac{2 \mathrm{EF}_{\mathrm{x}}-\mathrm{EE}_{\mathrm{y}}-\mathrm{FE}_{\mathrm{x}}}{2\left(\mathrm{EG}-F^{2}\right)}$, $\Gamma_{22}^{1}=\frac{2 \mathrm{GF}_{\mathrm{y}}-\mathrm{GG}_{\mathrm{x}}-\mathrm{FG}_{\mathrm{y}}}{2\left(\mathrm{EG}-F^{2}\right)}, \Gamma_{22}^{2}=\frac{\mathrm{EG}_{\mathrm{y}}-2 \mathrm{FF}_{\mathrm{y}}+\mathrm{FG}_{\mathrm{x}}}{2\left(\mathrm{EG}-F^{2}\right)}, \Gamma_{12}^{1}=\frac{\mathrm{GE}_{\mathrm{y}}-\mathrm{FG}_{\mathrm{x}}}{2\left(\mathrm{EG}-F^{2}\right)}$, $\Gamma_{12}^{2}=\frac{\mathrm{EG}_{\mathrm{x}}-\mathrm{FE}_{\mathrm{y}}}{2\left(\mathrm{EG}-F^{2}\right)}$. The subscript of $E, F, G$ represents the firstorder derivative with respect to $x$ or $y$. The Christoffel symbols $\Gamma_{11}^{1}, \Gamma_{12}^{1}, \Gamma_{22}^{1}, \Gamma_{11}^{2}, \Gamma_{12}^{2}, \Gamma_{22}^{2}$ only depend on the first fundamental coefficients $E, F, G$ and their derivatives.

Previous research has shown that different combinations of the equations in the equation set (3.3) can bring about different results. Moreover, studies also indicate that numerical problems will arise when the third equation in Gauss equations is included in HASM (Yue 2011). The best combination with the least error is:

$\left\{\begin{array}{l}f_{\mathrm{xx}}=\Gamma_{11}^{1} f_{\mathrm{x}}+\Gamma_{11}^{2} f_{\mathrm{y}}+\frac{L}{\sqrt{E+G-1}} \\ f_{\mathrm{yy}}=\Gamma_{22}^{1} f_{\mathrm{x}}+\Gamma_{22}^{2} f_{\mathrm{y}}+\frac{N}{\sqrt{E+G-1}}\end{array}\right.$

Let $\quad\left\{\left(x_{i}, y_{j}\right) \mid 0 \leq i \leq I+1,0 \leq j \leq J+1\right\}$ be the computational grids and $h$ be the grid size in $x$ and y directions. Finite difference methods are used for solving these differential equations. Equations (3.4) can be approximated as:

$$
\left\{\begin{array}{l}
\frac{f_{i+1, j}^{n+1}-2 f_{i, j}^{n+1}+f_{i-1, j}^{n+1}}{h^{2}}=\left(\Gamma_{11}^{1}\right)_{i, j}^{n} \frac{f_{i+1, j}^{n}-f_{i-1, j}^{n}}{2 h} \\
+\left(\Gamma_{11}^{2}\right)_{i, j}^{n} \frac{f_{i, j+1}^{n}-f_{i, j-1}^{n}}{2 h}+\frac{L_{i, j}^{n}}{\sqrt{E_{i, j}^{n}+G_{i, j}^{n}-1}} \\
\frac{f_{i, j}^{n+1}-2 f_{i, j}^{n+1}+f_{i, j-1}^{n+1}}{h^{2}}=\left(\Gamma_{22}^{1}\right)_{i, j}^{n} \frac{f_{i+1, j}^{n}-f_{i-1, j}^{n}}{2 h} \\
+\left(\Gamma_{22}^{2}\right)_{i, j}^{n} \frac{f_{i, j+1}^{n}-f_{i, j-1}^{n}}{2 h}+\frac{N_{i, j}^{n}}{\sqrt{E_{i, j}^{n}+G_{i, j}^{n}-1}}
\end{array}\right.
$$

In which $n$ represents the number of iterations.

Equations (3.5) can be transferred to the following form:

$\left\{\begin{array}{l}A x^{n+1}=d^{n} \\ B x^{n+1}=q^{n}\end{array}\right.$

In which $x^{n+1}=\left(f_{1,1}^{n+1}, \ldots, f_{1, J}^{n+1}, f_{2,1}^{n+1}, \ldots, f_{2, J}^{n+1}, \ldots, f_{I-}\right.$ $\left.{ }_{1,1}^{n+1}, \ldots, f_{I-1, J}^{n+1}, f_{I, 1}^{n+1}, \ldots, f_{I, J}^{n+1}\right)^{T}$

$A$ and $B$ denote the coefficient matrices of Eqs. (3.5) and $d, q$ are the right-hand vectors of Eqs. (3.5), respectively.
The boundary value of HASM is obtained by other surface modeling methods.

Equations (3.4) cannot determine unique solution function, in general, provided $f$ is sufficiently smooth. To single out a particular solution, value $x$ of solution function must be specified at some point $(x, y)$. The following equalityconstrained least squares problem is developed to make the interpolated values equal to or approximate to the sampled values at the sampling points.

$$
\left\{\begin{array}{c}
\min \left\|\left[\begin{array}{l}
A \\
B
\end{array}\right] x^{n+1}-\left[\begin{array}{l}
d \\
q
\end{array}\right]^{n}\right\|_{2} \\
S x^{n+1}=k
\end{array}\right.
$$

In which $S(l,(i-1) \cdot J+j)=1, \quad x(l)=k(l)$, this means that the sampled value is $k(l)$ at the $l$ sampling point $\left(x_{i}, y_{j}\right)$. However, when the sampling point deviates from the grid point, it is necessary to find the nearest grid point for which Taylor expansion is used to give an approximate value of this point. $\|$.$\| is the L2-norm of a vector.$

One way of obtaining an approximate solution to (3.7) is to solve the unconstrained least squares problem:

$\min _{x}\left\|\left[\begin{array}{c}A \\ B \\ \lambda S\end{array}\right] x^{n+1}-\left[\begin{array}{c}d \\ q \\ \lambda k\end{array}\right]^{n}\right\|$

For a Lagrange parameter $\lambda$ (Golub and Van Loan 2009). This problem (3.8) is equivalent to the symmetric positive definite linear system:

$W x^{n+1}=v^{n}$

In which $W=A^{\mathrm{T}} A+B^{\mathrm{T}} B+\lambda^{2} S^{\mathrm{T}} S, v=A^{\mathrm{T}} d+B^{\mathrm{T}-}$ $q+\lambda^{2} S^{\mathrm{T}} k, \lambda$ is the weight of the sampling points. For large values of $\lambda$, however, numerical problems arise (Golub and Van Loan 2009). Chen and Yue (2010) have stated that a smaller value of $\lambda$ is selected in a complex region and a bigger value is selected in a flat region. Therefore, for a specific application, we must adjust this parameter until a reasonable agreement is attained between model output and field measurement.

The version of HASM currently used takes into account only the first two equations of Gauss equations, without considering the mixed derivatives $f_{\mathrm{xy}}=\Gamma_{12}^{1} f_{\mathrm{x}}+\Gamma_{12}^{2} f_{\mathrm{y}}+$ $\frac{M}{\sqrt{E+G-1}}$ of this equation set (Yue 2011), although previous research (Yue and Du 2006) has considered the third equation in Gauss equations, the operation overflow. In this research, this equation is reconsidered into HASM to give a modified version of it, which first ensures that HASM is integral theoretically. 
The discrete equations of the modified HASM are then as follows:

$$
\left\{\begin{array}{l}
\frac{f_{i+1, j}^{n+1}-2 f_{i, j}^{n+1}+f_{i-1, j}^{n+1}}{h^{2}}=\left(\Gamma_{11}^{1}\right)_{i, j}^{n} \frac{f_{i+1, j}^{n}-f_{i-1, j}^{n}}{2 h} \\
+\left(\Gamma_{11}^{2}\right)_{i, j}^{n} \frac{f_{i, j+1}^{n}-f_{i, j-1}^{n}}{2 h}+\frac{L_{i, j}^{n}}{\sqrt{E_{i, j}^{n}+G_{i, j}^{n}-1}} \\
\frac{f_{i, j+1}^{n+1}-2 f_{i, j}^{n+1}+f_{i, j-1}^{n+1}}{h^{2}}=\left(\Gamma_{22}^{1}\right)_{i, j}^{n} \frac{f_{i+1, j}^{n}-f_{i-1, j}^{n}}{2 h} \\
+\left(\Gamma_{22}^{2}\right)_{i, j}^{n} \frac{f_{i, j+1}^{n}-f_{i, j-1}^{n}}{2 h}+\frac{N_{i, j}^{n}}{\sqrt{E_{i, j}^{n}+G_{i, j}^{n}-1}} \\
\frac{f_{i+1, j+1}^{n+1}-f_{i+1, j}^{n+1}-f_{i, j+1}^{n+1}+2 f_{i, j}^{n+1}-f_{i-1, j}^{n+1}-f_{i, j-1}^{n+1}+f_{i-1, j-1}^{n+1}}{2 h^{2}} \\
=\left(\Gamma_{12}^{1}\right)_{i, j}^{n} \frac{f_{i+1, j}^{n}-f_{i-1, j}^{n}}{2 h} \\
+\left(\Gamma_{12}^{2}\right)_{i, j}^{n} \frac{f_{i, j+1}^{n}-f_{i, j-1}^{n}}{2 h}+\frac{M_{i, j}^{n}}{\sqrt{E_{i, j}^{n}+G_{i, j}^{n}-1}}
\end{array}\right.
$$

Then, the constraint equation about sample point information is added to Eqs. (3.10) and the formulation of the modified HASM can be expressed as:

$$
\left\{\begin{array}{c}
\min \left\|\left[\begin{array}{l}
A \\
B \\
C
\end{array}\right] x^{n+1}-\left[\begin{array}{l}
d \\
q \\
p
\end{array}\right]^{n}\right\| \\
S x^{(n+1)}=k
\end{array}\right.
$$

where $A, B, C$ is the left-hand side of Eqs. (3.10), respectively. The structure of $A, B, S, k$ is the same as in HASM.

By introducing a Lagrange parameter $\lambda$, we finally get the linear equation of the modified HASM:

$W x^{n+1}=V^{n}$

In which, $W=A^{T} A+B^{T} B+C^{T} C+\lambda^{2} S^{T} S ; \quad V=A^{T-}$ $d+B^{T} q+C^{T} p+\lambda^{2} S^{T} k ; x$ is a vector that each element denotes the simulated value of the corresponding grid point.

Thus, the process of construct DEMs using the modified HASM can be divided into four steps:

Building the matrix $A, B, C$ according to the study area and resolution, building the matrix $S$ and vector $V$ according to the sample information, including the value and position of each sample point.

Building the large-scale sparse group of linear equations according to (3.12), the default value of $\lambda$ is 1 .

1 Solving the large-scale sparse group of linear equations, Preconditioned Conjugate-Gradient Technique (PCG) is exploited to solve the linear equations (Golub and Van 2009).

2 Output the solution of the equations according to the specific data format.
To be clear, the modified HASM does not require driving field anymore. Driving field provides initial value of the study area and is used to compute the first and the second fundamental coefficients in the former HASM.

Parallel algorithm designation

As discussed above, the modified HASM achieves a better simulation result, but at the same time it is more time consuming. For example, an area of $500 \times 500$ simulation requires more than $100 \mathrm{~s}$. So, it cannot be applied in a practical problem directly, and then the parallel version of the modified HASM is needed to apply it in a real surface simulation. We also found that the sampling rate and study scale are the most important effect factors in simulation efficiency, and this influence is different in different spatial positions. So the designation of the parallel algorithm should consider all these traits.

In digital terrain analysis and environmental science, many local terrain parameters' algorithms are designed based on data segmentation (Gong and Xie 2009; Song et al. 2013). The basic technique of these parallel algorithms involves segmenting the study area into small patches based on the process numbers, and then every process responding to a small patch and carrying out parameter calculation. Finally, a process is assigned to collect all the calculation results and write them to the existed file.

However, this parallel strategy is unsuitable to the modified HASM's parallelization for the following reasons. Firstly, there are no sufficient processes for research areas which are too large; and secondly even if we redivide the small patch into smaller ones, some smaller patches may consume more time than others, leading to a long total simulation time and computation resources being wasted, because all the other processes have to wait for the one that is not yet finished.

In parallel computation, the master-slave mode consists of two entities: master and multiple slaves. The master is responsible for decomposing the problem into small tasks (and distributes these tasks among a farm of slave processes), as well as for gathering the partial results to produce the nal result of the computation. The slave processes execute in a very simple cycle: get a message with the task, process the task, and send the result to the master. Usually, the communication takes place only between the master and the slaves (Hansen 1993; Yang et al. 2013).

So, this paper employs the master-slave mode to design the parallel algorithm based on the previous section regarding the characteristic of the modified HASM. Firstly, divide the study area into small patches on the basis of a given patch size (for example, $100 \times 100$ or $200 \times 200$ ), and then assign each small patch to a computation process. When one small process finishes the current simulation, a 
new small patch will be assigned to it from the ones left until all the small patches are simulated successfully. To make the simulation convenient and orderly, a process (for example 0 ) is appointed as the main process which is responsible for assigning tasks to other processes and saving the simulation results. The total chart flow of the parallel algorithm is shown in Fig. 2.

\section{Results and discussion}

Accuracy and computation speed analysis under data segmentation

As discussed in the introduction, all the HASM algorithms take the study area as a unity and construct a large-scale system of linear equations, excluding the HASM-AC algorithm, which is dependent on a driving field. As for the

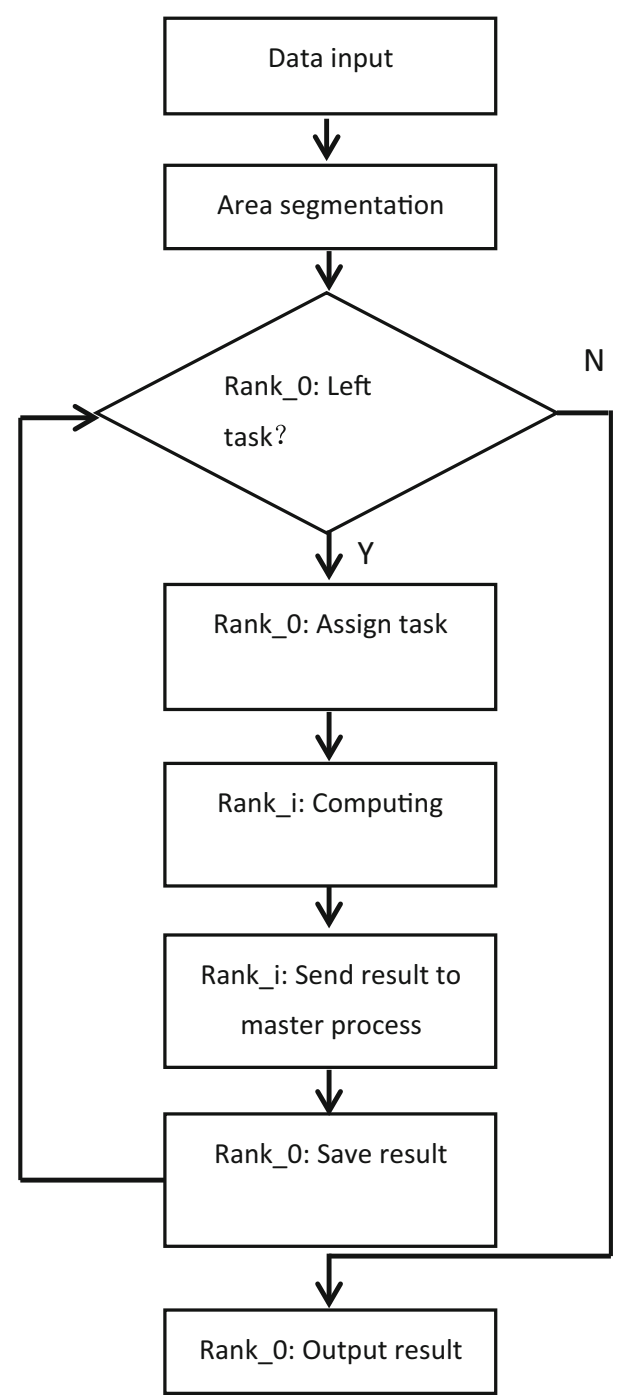

Fig. 2 Flow chart of the parallel algorithm modified HASM, adopting the third Gaussian equation makes the HASM theoretically perfect, and also introduces more computation. For the DEM generation, in particular, large computation and computer memory demand make it impossible to solve the question via the usual method. So in this paper, an area segmentation-based HASM algorithm is proposed and realized to satisfy the large area and highresolution computation. The so-called area segmentation strategy divides the study area into many small patches, and then computes each patch in order, before lastly gathering all the results of the patches to obtain the solution of the entire problem.

To ensure the modified HASM simulation accuracy in DEM generation, this paper first analyses simulation accuracy under an area segmentation strategy before designing a parallel version of the modified HASM. In addition, it is important to select a proper segmentation particle size when designing a parallel algorithm based on area-segmentation, as a larger particle size can lead to too much time being spent on computing each small patch area, while a smaller particle size can increase the time of data communication between different processes. Thus, the time consumption of different particle sizes is discussed in this section.

\section{Simulation accuracy analysis}

Ten test areas are selected randomly and consider the terrain features (Fig. 1). For each test area, ten tests are designed according to different spatial sampling rates, i.e., 5, $4,3,2,1,0.9,0.8,0.7,0.6,0.5,0.4,0.3,0.2$, and $0.1 \%$, respectively. To compare simulation accuracy both with the old HASM and classical interpolation methods (taking Kriging as an example), in this paper each test was performed three times using different methods, the modified HASM, the old HASM and ordinary Kriging. $0.5 \%$ points of the origin Lidar points were selected as the validation points for each test area, so the error of the simulation result compared with the true value could be computed at every validation point. This paper computes the mean absolute error (MAE) and the root mean square error (RMSE) for each test, and then the mean values of the 10 areas are computed, respectively, as the mean absolute error and the standard deviation under a certain sampling rate. Figure 3 shows the simulation accuracy variation as the sampling rate decreases clearly.

It is obvious that the simulation error of the three methods all decreased as the sample rate increased, from 0.1 to $5 \%$. However, the modified HASM got the best simulation result at a low sample rate, particularly, at the $0.1 \%$ sample rate, where the modified HASM showed a significant improvement compared to the old HASM and ordinary Kriging, with the mean absolute error and 

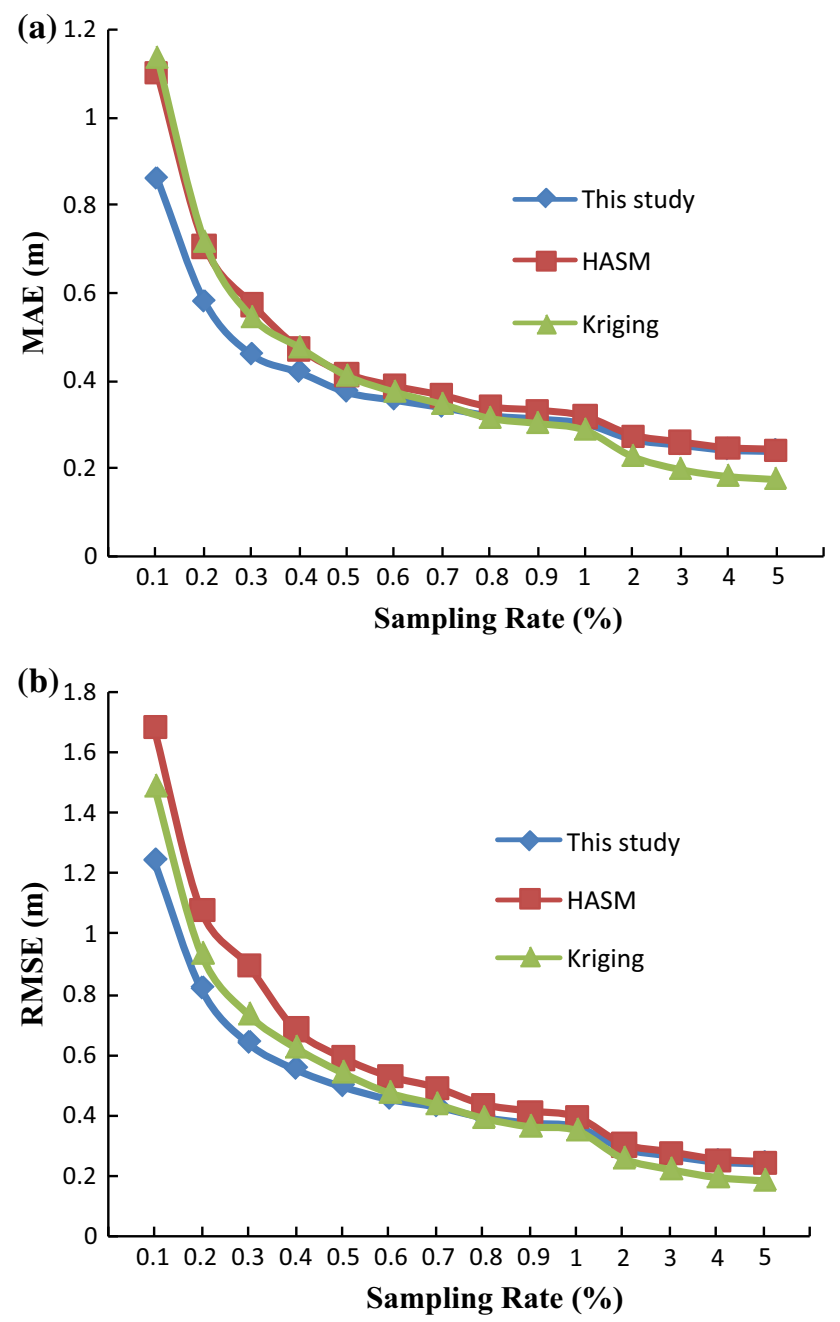

Fig. 3 Errors of the three methods at different sampling rates, a MAE, b RSME

standard deviation of the modified HASM at 0.8 and $1.2 \mathrm{~m}$, compared to the old HASM and ordinary Kriging at more than 1.1 and $1.5 \mathrm{~m}$.

This demonstrates that, compared with these other methods, the modified HASM attains better simulation results at a low sample rate. This gives the modified HASM a significant advantage in practical application, because on the one hand it is usually hard to obtain more sample points in practical problems; and on the other hand, scientific researches nowadays always require higher resolution DEMs, resulting in decreasing the sample rate further. With this in mind, this paper focuses on the simulation at low sample rate problem, and simulation accuracy analysis at the sample rate $0.1 \%$ is discussed in the next section.

Table 1 shows the largest error of the ten test areas of the three methods, and the advantages of the modified HASM are clearly shown. It is obviously apparent that the significant characteristic of the modified HASM is that it eliminates the huge error; that is to say, the error of the
Table 1 Max errors of the three methods in the 10 test areas $(\mathrm{m})$

\begin{tabular}{llcc}
\hline ID & This study & HASM & Kriging \\
\hline 1 & 3.54 & 7.53 & 11.23 \\
2 & 3.69 & 5.57 & 5.69 \\
3 & 1.38 & 3.11 & 5.26 \\
4 & 1.46 & 4.42 & 4.98 \\
5 & 3.55 & 7.15 & 12.27 \\
6 & 5.35 & 7.32 & 12.55 \\
7 & 8.33 & 13.68 & 16.34 \\
8 & 6.32 & 8.89 & 9.67 \\
9 & 6.55 & 7.68 & 9.53 \\
10 & 6.56 & 8.08 & 13.73 \\
\hline
\end{tabular}

modified HASM's simulation results is well distributed. For example, in the first test area, the largest error of the old HASM and ordinary Kriging is 7.53 and $11.23 \mathrm{~m}$, respectively, while the modified HASM is only $3.54 \mathrm{~m}$. To ensure the above conclusion make sense in the statistics, $T$ paired tests of the max errors are carried out between the modified HASM and the compared methods. Test results show that the modified HASM shows a significant superiority over the former HASM $(t=6.418$, sig. $<0.01)$ and Kriging Method $(t=6.858$, sig. $<0.01)$.

In the previous section, we initially used validation points to analyze the advantages of the modified HASM compared to the other methods. In this section, we will discuss the simulation analysis of the different methods with respect to digital terrain analysis. Slope is one of the most important terrain parameters that depict terrain morphology, and it is widely used in digital terrain analysis (Zhou and Liu 2004; Tang et al. 2008). So this research takes the slope as the accuracy index of the simulation results. The slope computed from the DEMs generated by the origin Lidar points is deemed as the true value, because the Lidar point's density is equivalent to a sampling rate of $11 \%$. There is little difference between DEMs produced by any method at this sampling rate and it can be taken as the real surface.

For each test area, the slope maps of real surface and the simulation results from the three methods are produced in ArcGIS 10.1, and then the mean slope values are computed (Fig. 4). Generally speaking, the slope value of the simulated surface is lower than the value of the real surface. This behavior is also reflected in this study, with all the simulated slope values of the three methods being lower than the real slope value. However, the slope computed from the modified HASM's simulation is much more in accordance with the real surface. So, it can be concluded that the modified HASM better simulates the surface with respect to terrain morphology.

The previous section mainly focused on comparing the simulation accuracy of the modified HASM and other 


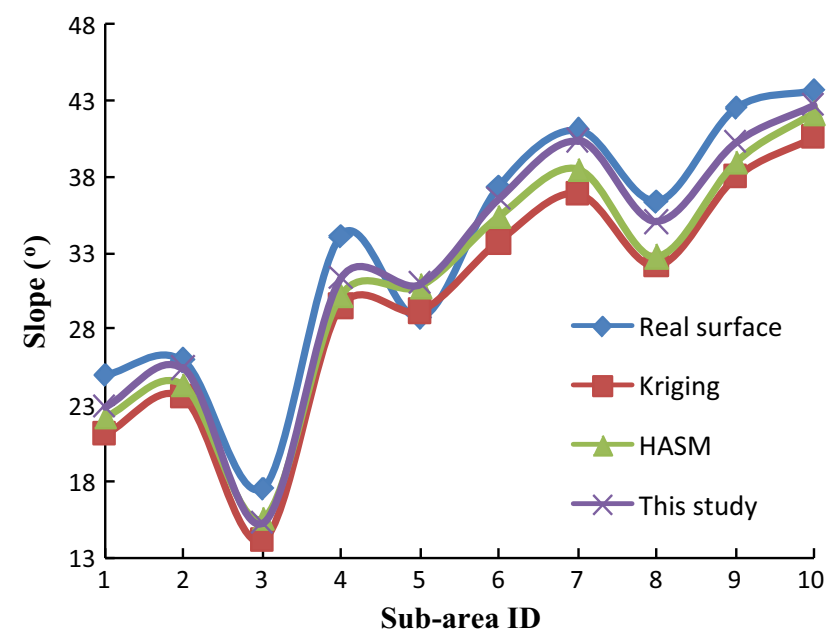

Fig. 4 Slope comparison of the different methods in the 10 test areas
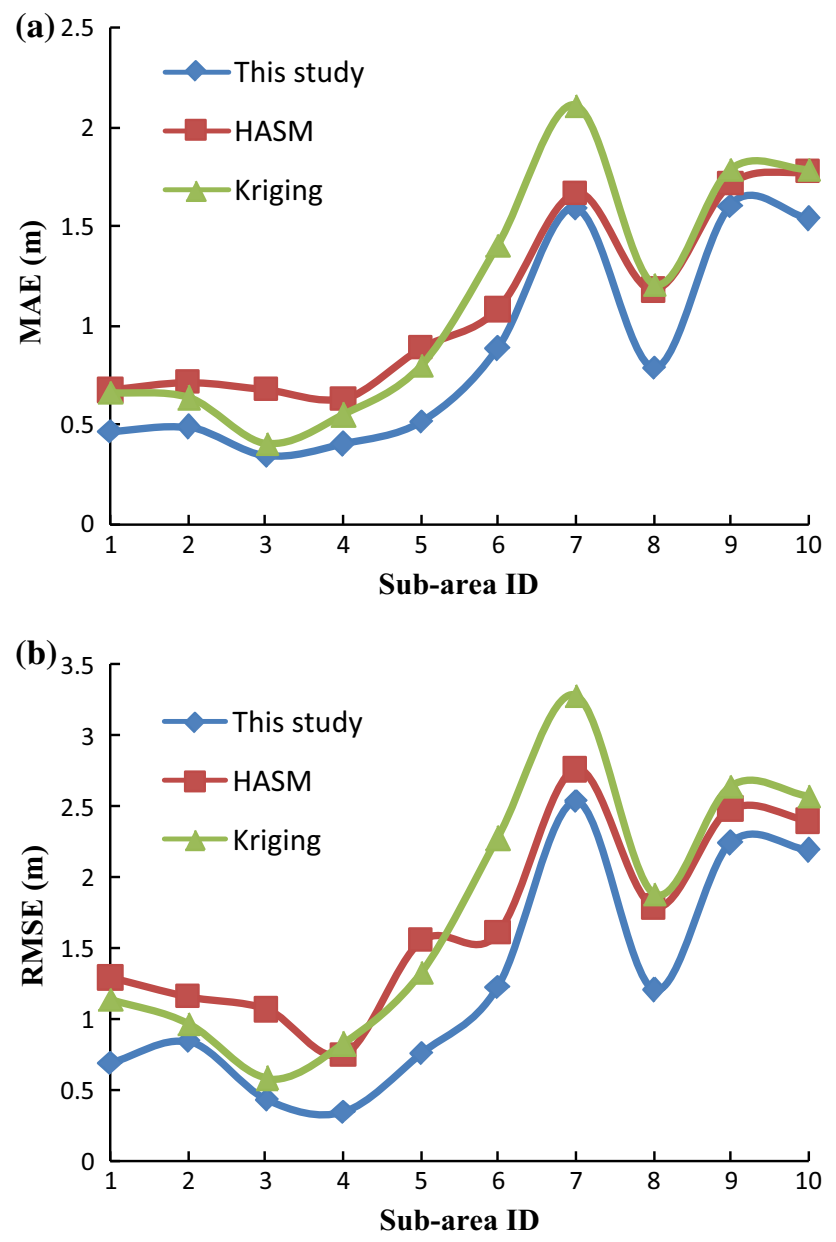

Fig. 5 Simulation error when the sampling rate is $0.1 \%$, a MAE, b RMSE

methods. In this section, the simulation accuracy of the modified HASM in different test areas will be discussed, because it is very important in helping us to understand

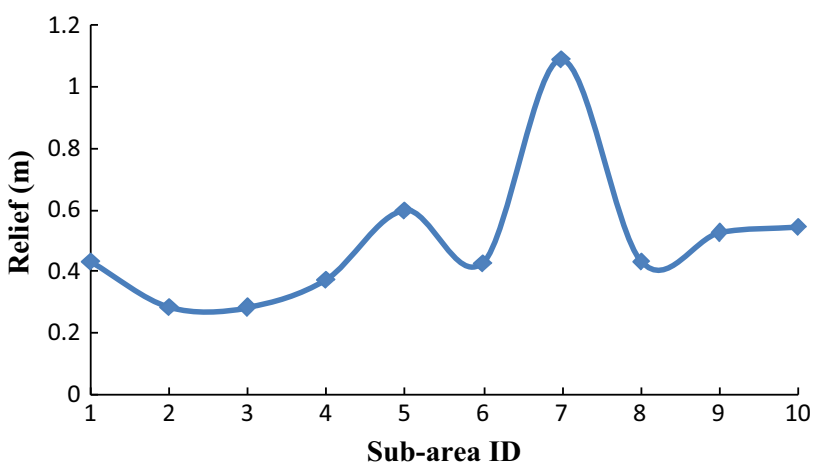

Fig. 6 Relief of the 10 test areas

how to apply the modified HASM to practical problems. Figure 5 shows the error statistics of the ten test areas at a sampling rate of $0.1 \%$.

It can be seen that for every interpolation method, there is a significant difference between simulation accuracy in different areas. Though the modified HASM achieves better simulation accuracy, the variation trend of the errors among different test areas is generally in accordance with the other two methods. So it can be inferred that this variation of errors among different test areas is caused by the terrain surface itself. This paper calculates the relief of the ten test areas (Fig. 6). The so-called terrain's relief is an index that reflects the ups and downs of the surface, and is usually used to describe the complexity of the surface (Tu and Liu 1991). The relief index can be calculated as:

Relief $=H_{\max }-H_{\min }$

where $H_{\max }$ represents the maximum elevation in the study area, and $H_{\mathrm{min}}$ is the minimum elevation in the study area.

Comparing Fig. 6 with Fig. 5, it is apparent that the relief index is in consistent with the error in the different areas, meaning that the relief index can be used to explain the simulation variation in these areas. That is to say, a larger relief index may correspond with a larger simulation error, and vice versa. It can be easily interpreted because a large relief index indicates a complicated terrain, so a low sampling rate cannot depict the surface variation as well as in a flat area. A similar conclusion can be found in research previously carried out (Yue et al. 2010a).

\section{Time analysis}

We analyzed the simulation accuracy of the modified HASM in DEM's construction. However, as discussed in the introduction, the computation characteristic of HASM makes it more time consuming, and the modified HASM yet more so because it brings in the third Eq. (3.10). So, to design a good parallel algorithm version for practical application, special analysis of time consumption in the 


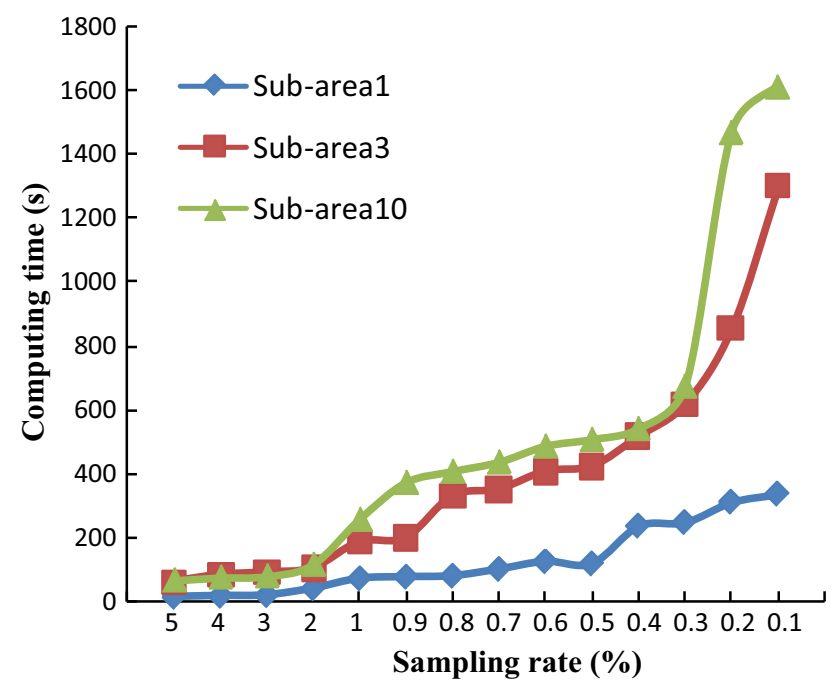

Fig. 7 Time consumption of different sampling rates

modified HASM and its influencing factors (such as sampling rate, study area scale, et al.) is necessary.

Figure 7 describes time consumption at different sampling rates in some test areas, and it can be found that sampling rates have a significant effect on time consumption, and that this effect is varied among different areas. For example, time consumption of test area 1, test area 2 and test area 3 is 4,5 , and $6 \mathrm{~s}$, respectively, when the sampling rate is $1 \%$; obviously there is no significant difference. Time consumption in the three test areas all go up while the sampling rate decreases, but the amplitude shows a significant difference. When the sampling rate finally reduces to $0.1 \%$, the time consumption of test area 1 , test area 2 and test area 3 is 22, 33 and 44 s, respectively.

Compared with the sampling rate, the study scale has a more obvious effect on time consumed because the study scale directly determines the scale of large linear system equations. This paper selects 10 test points in the study area randomly, and extracts five different test areas at each point: $100 \times 100,200 \times 200,300 \times 300,400 \times 400$ and $500 \times 500$. The origin Lidar points are used as the sample points which are equivalent to a sampling rate of $11 \%$. Figure 8 describes the average time consumed under a different study scale of the 10 test points, and shows that the study scale has a linear effect on time consumption. In addition, time consumed under the same study scale at different points also produces different results. For example, when the study scale is $500 \times 500$, time consumption of test point 3 is $40 \mathrm{~s}$, while that of test point 2 is $58 \mathrm{~s}$.

\section{Parallel efficiency test}

The computing machine used in the study is IBM blade cluster system, and the compile software is $\mathrm{C}++12.0$. The

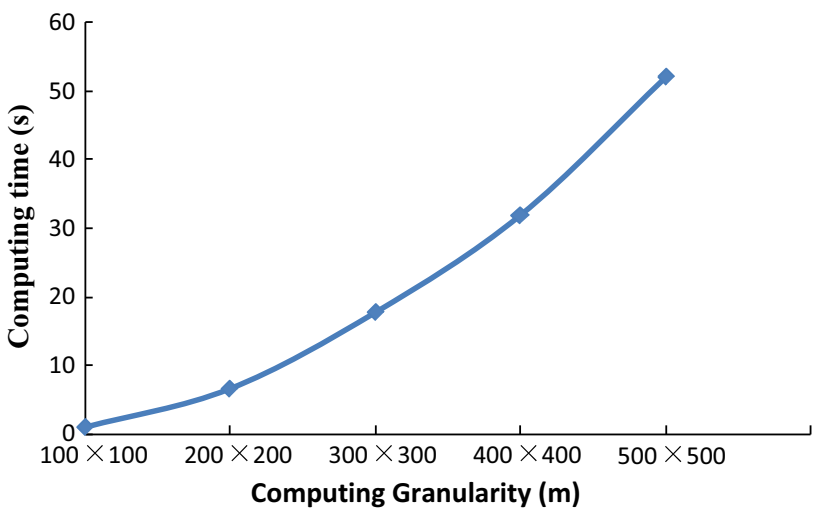

Fig. 8 Time consumption of different computing granularity

test area for parallel efficiency is $2.5 \mathrm{~km} \times 2.5 \mathrm{~km}$ (Fig. 1), the resolution is also set to be $0.5 \mathrm{~m}$, and so the total study scale is $5000 \times 5000$. Three kinds of patch sizes are selected: $100 \times 100,200 \times 200$ and $300 \times 300$. Meanwhile, the sampling rate is also considered because it significantly influences on the simulation efficiency, and so we analyze the computing efficiency for sampling rates of $1 \%$.

Statistics of time consumption and the speed-up ratio of each computing granularity are shown in Fig. 9. Of which the speed-up ratio is calculated as:

$S_{\mathrm{p}}(q)=\frac{T_{\mathrm{s}}}{T_{\mathrm{p}}(q)}$

where $q$ represents the process number, $T_{\mathrm{s}}$ is the serial time elapsed, and $T_{\mathrm{p}}(q)$ is the parallel time elapsed when the process number is $q$.

It can be seen that for each sampling rate, the variation of time consumption and speed-up ratio as the processes increase is very similar. When the computing granularity is $100 \times 100$, the computing time is small, but this computing granularity also gets a lower speed-up ratio because a small computing granularity leads to more small patches and requires more communication between the slave process and master process. Therefore, too much communication makes a lower speed-up ratio.

Generally speaking, the parallel efficiency is satisfied and acceptable. The computing time for a $5000 \times 5000$ area is limited to $1500 \mathrm{~s}$ while the current serial algorithm cannot perform the same work. The speed-up ratio is larger than five when the process number is 10 , which means that the parallel efficiency exceeds 0.5 .

\section{Conclusion}

This paper shows that when HASM is applied to surface simulation, it improves the simulation accuracy compared 


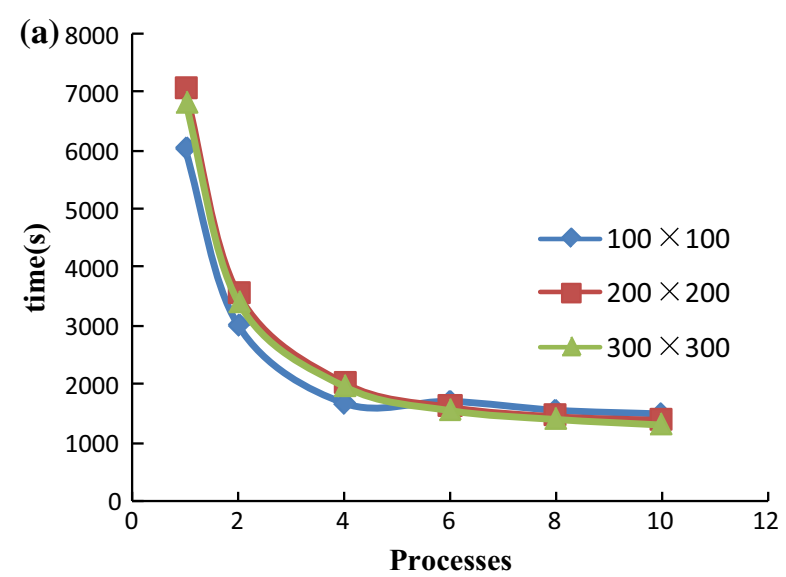

(b)

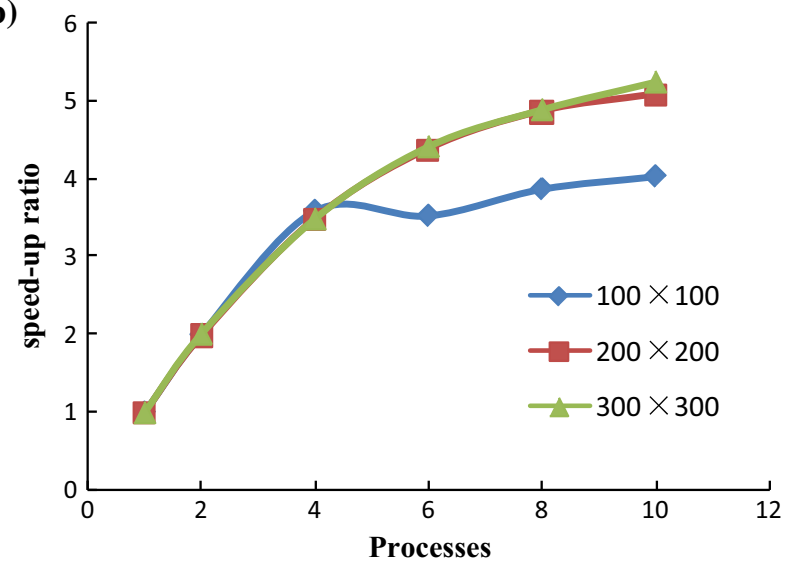

Fig. 9 Time consumption: $\mathbf{a}$ and speed-up ratio $\mathbf{b}$ when the sampling rate is $1 \%$

to the classical spatial interpolation methods, such as IDW, Kriging and so on. However, HASM also exhibits certain weaknesses, two of which are serious. The first is that HASM requires driving fields to run the model, which means that HASM cannot perform surface simulation solely based on sample points. The second is that HASM consumes so much time and memory that it is impossible to employ as a method of solving large-scale problems when performing spatial Interpolation. The primary purpose of this paper is to resolve these defects and indeed make HASM useful in large-scale problems.

The key contribution of this research is to bring in the third equation to make HASM more theoretically perfect and realize the parallel version of the modified HASM. This paper also aims to resolve the accuracy and efficiency problem in the DEMs construction. Tests show that the modified HASM can simulate the surface with high accuracy independently and the parallel version makes it possible to solve large area and high-resolution simulation. Since HASM is not only used in DEM construction but also widely used in other ecology fields (Zhao et al. 2014b; Yue et al. 2014), and lower simulation efficiency also creates problems in these other applications, it makes sense for subsequent work to focus on parallel algorithm research for the sparse sample point problem.

Acknowledgments This work is supported by National Natural Science Foundation of China (91325204), by Independent Research Project funded by State Key Laboratory of Resources and Environment Information System, by National High-tech R\&D Program of the Ministry of Science and Technology of the People's Republic of China (2013AA122003), by National Key Technologies R\&D Program of the Ministry of Science and Technology of the People's Republic of China (2013BACO3B05) and by National Basic Research Priorities Program (2010CB950904) of Ministry of Science and Technology of the People's Republic of China.

\section{References}

Alkhasawneh MS, Ngah UK, Tay LT, Isa NAM (2014) Determination of importance for comprehensive topographic factors on landslide hazard mapping using artificial neural network. Environ Earth Sci 72:787-799

Bragard L (1965) Method to determine the shape of the topographical earth surface by means of gravity measurements on that surface by solving two integral equations. Stud Geophys Geod 9(2): 108-112

Chen CF, Yue TX (2010) A method of DEM construction and related error analysis. Comput Geosci 36:717-725

De Gbaaff-Hunter J (1937) The shape of the Earth's surface expressed in terms of gravity at ground level. Bull Géodésique 56(1):191-200

Dech S (2005) The Earth surface In: Feuerbacher B, Stoewer H (eds) Utilization of space, Bonn, Springer pp 53-90

Golub GH, Van Loan CF (2009) Matrix computations. Beijing, Posts and Telecom Press

Gong JY, Xie JB (2009) Extraction of drainage networks from large terrain datasets using high throughput computing. Comput Geosci 35(2):337-346

Gupta M, Mohanty KK, Kumar D, Banerjee R (2014) Monitoring surface elevation changes in Jharia coalfield, India using synthetic aperture radar interferometry. Environ Earth Sci 71:2875-2883

Hansen PB (1993) Model programs for computational science: a programming methodology for multicomputers. Concurrency: practice and experience, 5(5):407-423

Henderson DW (1998) Differential Geometry. Prentice-Hall, London

Ionescu IR, Volkov D (2008) Earth surface effects on active faults: an eigenvalue asymptotic analysis. J Comput Appl Math 220(1-2):143-162

Kerimov IA (2009) F-approximation of the Earth's surface topography. Izvestiya Phys Solid Earth 45:719-729

Liang B, Di L, Zhao CY, Peng SZ, Peng HH, Wang C, Wang Y, Liu YY (2013) Altitude distribution of Aboverround biomass of typical shrubs in the Tianlaochi watershed of Qilian mountains. Acta Agrestia Sinica 21(4):664-669 (in Chinese with English abstract)

Murray AB, Lazarus E, Ashton A, Baas A, Coco G, Coulthard T, Fonstad M, Haff P, McNamara D, Paola C, Pelletier J, Reinhardt L (2009) Geomorphology, complexity, and the emerging science of the Earth's surface. Geomorphology 103(3):496-505

Niu WY (1993) Some theoretical conclusions of morphological systems on the Earth's surface. Quat Sci 13(2):129-141 (in Chinese with English abstract)

Petrovskaya MS (1977) Generalization of Laplace's expansion to the Earth's surface. J Geodesy 51(1):53-62 
Somasundaram D (2005) Differential geometry. Alpha Science International Ltd, Harrow

Song XD, Tang GA, Li FY, Jiang L, Zhou Y, Qian KJ (2013) Extraction of loess shoulder-line based on the parallel GVF snake model in the loess hilly area of China. Comput Geosci 52:11-20

Stepanova IE (2007) S-Approximation of the Earth's surface topography. Izvestiya Phys Solid Earth 43(6):466-475

Strakhov VN, Kerimov IA, Strakhov AV (1999) Linear analytical approximations of the Earth's surface topography In: Proceedings of the 1st All-Russia conference on geophysics and mathematics. OIFZ RAN, Moscow pp 198-212 (in Russian)

Tang GA, Li FY, Liu XJ, Long Y, Yang X (2008) Research on the sloe spectrum of the Loess Plateau. Sci China Ser E: Technol Sci 51(Supp. 1):175-185

Tang CL, Hu JC, Lin ML, Yuan RM, Cheng CC (2013) The mechanism of the 1941 Tsaoling landslide, Taiwan: insight from a 2D discrete element simulation. Environ Earth Sci 70:1005-1019

Tarolli P, Arrowsmith JR, Vivoni ER (2009) Understanding earth surface processes from remotely sensed digital terrain models. Geomorphology 113(1-2):1-3

Toponogov VA (2006) Differential geometry of curves and surfaces. Birkhaeuser Boston, New York

Tu HM, Liu ZD (1991) Study on relief amplitude in China. Acta Geodaetica Cartogr Sin 20(11):311-319 (in Chinese with English abstract)

Yang Y, Wu JF, Sun XM, Wu JC, Zheng CM (2013) Development and application of a master-slave parallel hybrid multi-objective evolutionary algorithm for groundwater remediation design. Environ Earth Sci 70:2481-2494

Yue TX (2011) Surface modelling: high accuracy and high speed methods. CRC Press, New York
Yue TX, Du ZP (2006) Numerical test for optimum formulation of high accuracy surface modeling. Geo-Inform Sci 8:83-87 (in Chinese with English abstract)

Yue TX, Song YJ (2008) The YUE-HASM Method. In: Deren Li, Yong Ge, Giles M. Foody (eds) Accuracy in Geomatics, pp $148-153$

Yue TX, Wang SH (2010) Adjustment computation of HASM: a high-accuracy and high-speed method. Int J Geogr Inf Sci 24:1725-1743

Yue TX, Du ZP, Song DJ, Gong Y (2007) A new method of surface modeling and its application to DEM construction. Geomorphology 91:161-172

Yue TX, Chen CF, Li BL (2010a) An adaptive method of high accuracy surface modeling and its application to simulating elevation surfaces. Trans GIS 14:615-630

Yue TX, Song DJ, Du ZP, Wang W (2010b) High-accuracy surface modelling and its application to DEM generation. Int J Remote Sens 31:2205-2226

Yue TX, Zhao MW, Zhang XY (2014) A high-accuracy method for filling voids of remotely sensed $\mathrm{CO} 2$ surfaces and its verification, J Clean Prod (in press)

Zhao N, Yue TX, Zhao MW, Du ZP, Chen CF (2014a) Sensitivity studies of a high accuracy surface modeling method. Sci China Earth Sci 57(1):1-11

Zhao MW, Yue TX, Zhao N, Sun XF, Zhang XY (2014b) Combining LPJ-GUESS and HASM to simulate the spatial distribution of forest vegetation carbon stock in China. J Geog Sci 24(2):249-258

Zhou QM, Liu XJ (2004) Analysis of errors of derived slope and aspect related to DEM data properties. Comput Geosci 30:369-378 